\section{P120 Draw Your Dinner: Examining Perceptions of Meal Size Using Incrementally Reduced Plate Sizes in College Students}

David Sharp, PhD, Dsharp7@kent.edu, Kent State University, 135 Nixson Hall, Kent, OH 44240; Jennifer St Peter, BS, University of Minnesota Medical School

Background (Background, Rationale, Prior Research, and/or Theory): The ways in which food is presented can influence the amount of food selected and consumed as well as how accurately those meals are perceived and recalled. Hence, if the method of food recall is modified through the use of incrementally changing plate sizes for participants to draw their meals on, they may align their food sizes to fit the plate rather than accurately reporting portion sizes.

Objective: The purpose of this study was to examine the association between plate size and meal recall through drawn food representations over a 10-week period.

Study Design, Setting, Participants, Intervention: Using a longitudinal design, we had 293 undergraduate students enrolled in an introductory Nutrition lecture course draw their last night's meal for 10 weeks on either a 10.5" diameter plate, an 8.5" diameter plate, or a 10.5" diameter plate that was reduced in size by .2 inches every week. One hundred ninety-five students who completed $>60 \%$ of their meal recalls, including the first and final weeks, were included for analysis.

Outcome Measures and Analysis: One-way and twoway ANOVA as well as independent samples t-tests were used to compare total area, \% of plate coverage, individual food group food portion size across Large, Small, and Incrementally-changing plates.

Results: Participants provided with 10.5" diameter plates generally drew significantly more food on them than participants with 8.5 " diameter plates $(P=<.001)$. Participants provided with incrementally shrinking plates drew meal sizes similar to the large plates at week 1 and drew meal sizes similar to small plates by week 10 .

Conclusions and Implications: These findings suggest that people's conceptualization of previous meal consumption may be influenced by the size of the plate on which they draw upon, suggesting that the external environment can modify participant drawn recall of their meal sizes.

Funding: None.

\section{P121 Effects of Time Restricted Feeding on Weight Loss and Sleep: A Randomized, Cross-0ver Trial}

A'nna Sewall, BS, RDN, CPT, ams724@cornell.edu, Cornell University, Savage Hall Forest Home Drive, Ithaca, NY 14853; David Levitsky, PhD, BS;

Laura Barre, BS, RDN, MD

Background (Background, Rationale, Prior Research, and/or Theory): Time Restricted Feeding (TRF) is the practice of consuming all of one's calories within a restricted period of time in a day, and is gaining popularity as a method to promote weight loss and improve metabolic health and sleep. Evidence based research in humans is insufficient to make recommendations.

Objective: To examine the short-term effects of TRF on weight and sleep time and quality in adults.

Study Design, Setting, Participants, Intervention: A randomized, cross-over study of TRF was conducted. Adults $(n=60)$ were randomized to two weeks of TRF followed by two weeks of Control (CON) or vice versa. During both phases participants ate and drank ad libitum. Intake was restricted to a 12-hour period of time in the TRF phase and unrestricted in the CON phase.

Outcome Measures and Analysis: Daily weights were collected throughout using blue-tooth enabled scales and remote monitoring. Participants self-reported subjective sleep quality and physical activity (hours per week). Paired t-tests were used within-subjects to compare CON to TRF data.

Results: Participants were a mean age of 46.6 years ( $\mathrm{SD}=10$ years); 86\% Caucasian; 88\% women; and a mean BMI of $27.3 \mathrm{~kg} / \mathrm{m}$ 2.(SD = 5.56) For those in the TRF-CON group, weight change was $-0.13 \mathrm{lbs}(\mathrm{SD}=3.04$, $P=.98)$ and for those in the CON-TRF group -0.04 lbs $(\mathrm{SD}=3.01, P=.96)$. Subjective sleep quality was higher during the TRF vs. CON phase, $\mathrm{t}(58)=3.25, P=.001$. Physical activity was not significantly different between the two phases (219 $\mathrm{min}, \mathrm{SD}=1.87, P=.29$ ).

Conclusions and Implications: In adults, 2 weeks of TRF did not result in significant changes in weight, however sleep quality was better. TRF may be an advantageous lifestyle to improve perceived sleep quality in adults short term. Further research is necessary to determine the effects of TRF on body weight in free-living adults eating ad libitum longer term.

Funding: None.

\section{P122 Exploring the Importance of Healthfulness Among Food Truck Operators}

Melissa M. Reznar,PhD, MPH, reznar@oakland.edu, Oakland University, 433 Meadow Brook Road, 3102 Human Health Building, Rochester, MI 48309; Jamie Eathorne, MPH, Monroe County Health Department; Katherine Brennecke, BS, Oakland University; Brandon Metcalf, BS

Background (Background, Rationale, Prior Research, and/or Theory): Food trucks have become an increasingly popular part of the food scene, yet little research has investigated food truck operators or their offerings.

Objective: This study examines the characteristics of food truck operators in the Metropolitan Detroit area and their attitudes toward health and nutrition of food truck menu items.

Study Design, Setting, Participants, Intervention: Food truck operators were identified by searching state

Continued on page S66 